\title{
Density Variation During Respiration Affects PET Quantitation in the Lung
}

\author{
Beverley F. Holman, Student Member, IEEE, Vesna Cuplov, Ottavia Bertolli, Student Member, IEEE, \\ Ashley M. Groves, Brian F. Hutton, Senior Member, IEEE and Kris Thielemans, Senior Member, IEEE
}

\begin{abstract}
PET quantitation depends on the accuracy of the CT-derived attenuation correction map. In the lung, respiration leads to both positional and density mismatches, causing PET quantitation errors at lung borders but also within the whole lung. The aim of this work is to determine the extent of the associated errors on the measured time activity curves (TACs) and the corresponding kinetic parameter estimates. 5 patients with idiopathic pulmonary fibrosis underwent dynamic ${ }^{18}$ F-FDG PET and cine-CT imaging as part of an ongoing study. The cineCT was amplitude gated using PCA techniques to produce end expiration (EXP), end inspiration (INS) and mid-breathing cycle (MID) gates representative of a short clinical CT acquisition. The ungated PET data were reconstructed with each CT gate and the TACs and kinetic parameters compared. Patient representative XCAT simulations with varying lung density, both with and without motion, were also produced to represent the above study allowing comparison of true to measured results. In all cases, the obtained PET TACs differed with each CT gate. For ROIs internal to the lung, the effect was dominated by changes in density, as opposed to motion. The errors in the TACs varied with time, providing evidence that errors due to attenuation mismatch depend on activity distribution. In the simulations, some kinetic parameters were over- and under-estimated by a factor of 2 in the INS and EXP gates respectively. For the patients, the maximum variation in kinetic parameters was $20 \%$. Our results show that whole lung density changes during the respiratory cycle have a significant impact on PET quantitation. This is especially true of the kinetic parameter estimates as the extent of the error is dependent on tracer distribution which varies with time. It is therefore vital to use matched PET/CT for attenuation correction.
\end{abstract}

Index Terms-Lung, Density, Respiration, PET-CT, Attenuatuion Correction.

\section{INTRODUCTION}

$\mathbf{T}$ O obtain quantitative PET images, accurate attenuation correction (AC) maps are required, and this is known to be difficult when imaging in the thorax due to respiratory motion. The causes for respiratory AC mismatches can be separated in 2 types: motion, leading to location mismatch, and density variations, due to lung fractional air volume change over the course of the breathing cycle. Motion correction

B. Holman, V. Cuplov, O. Bertolli, A. Groves, B. Hutton and K. Thielemans are with the Institute of Nuclear Medicine, UCLH, 235 Euston Road (T-5), London, NW1 2BU, UK. e-mail: beverley.holman.12@ucl.ac.uk

B. Hutton is also with the Centre for Medical Radiation Physics, University of Wollongong, Australia

We acknowledge funding support from GlaxoSmithKline (STU100028576), General Electric Healthcare and the Engineering and Physical Sciences Research Council (EP/K0052781/1 and 13220093). Also, this project is supported by researchers at the National Institute for Health Research, University College London Hospitals Biomedical Research Centre. techniques have been widely explored in the literature, however, little attention has been given to the density mismatches. Previous work has shown that errors in the reconstructed PET images associated with these attenuation mismatches are mostly local to the region where the attenuation mismatch occurs, but will depend on the tracer distribution around the region of the mismatch, i.e. in the entire thorax [1]. As tracer distribution is variable in dynamic imaging, this work aims to determine the effect of attenuation mismatches on measured time activity curves (TACs) and their associated estimated kinetic parameters.

\section{Methods}

Patient acquisition and simulations were used to determine the variation in ungated PET TACs and kinetic parameter estimates when using $\mathrm{AC}$ based on 3 different CT gates representing end inspiration (INS), end expiration (EXP) and mid-stage of the breathing cycle (MID) to investigate the effect of using breath-hold CT for AC.

For the patient data, 5 patients with diagnosed idiopathic pulmonary fibrosis (IPF) undergoing ${ }^{18} \mathrm{~F}$-FDG dynamic PET/CT were used. These patients were imaged supine, immediately following injection, using a GE Discovery VCT PET/CT scanner [2]. The study protocol is outlined in table I.

Noise free simulations were performed to allow study of the attenuation mismatch effect size. The XCAT phantom [3] was used to replicate the study in table I by measuring the TACs in all organs of a single patient chosen at random. As well as the three CT gates described above, an average CT (AVE) was created to represent the 'True' attenuation map for reconstructing ungated PET data. While the XCAT phantom accounts for motion, it does not adjust the lung values for density changes. Therefore, the 4 maps were edited such that the density varied linearly with whole lung volume. This density change was also incorporated to change the PET activity concentration, as motivated by work on the 'Tissue Fraction Effect' (TFE) [4], [5]. The lung fractional air volume was determined from the AC maps (as previously described by Lambrou et al) and used to correct the PET accordingly.

Two simulation sets were created, the first with motion and density change as would be expected in a patient and the second with density change only (no motion) to allow differentiation from motion-only errors.

The PET data underwent FBP reconstruction with added scatter and true attenuation map equivalent to the AVE for the motion and density case and MID for the density change 
TABLE I

PATIENT ACQUisition Protocol.

\begin{tabular}{cl}
\hline Time Post Injection & Acquisition \\
\hline & Cine CT (1 PET bed) \\
Omins & Patient Injection \\
0mins & 20min Dynamic PET (1 bed) \\
& $6 \times 10 \mathrm{~s}, 3 \times 20 \mathrm{~s}, 3 \times 60 \mathrm{~s}, 5 \times 120 \mathrm{~s}, 1 \times 300 \mathrm{~s}$ \\
\hline
\end{tabular}

TABLE II

VARIATION IN CONCENTRATION ERROR OVER TIME FOR THE MOTION AND DENSITY AND DENSITY ONLY SIMULATIONS. THE MAXIMUM ERROR WAS LOCATED IN THE TAC PEAK IN ALL CASES AND THE MINIMUM AT THE FINAL TIME POINT.

\begin{tabular}{lcccc}
\hline & \multicolumn{2}{c}{ Motion and Density } & \multicolumn{2}{c}{ Density Only } \\
\hline & Peak & Final Time Point & Peak & Final Time Point \\
\hline EXP & $-7.2 \%$ & $-1.1 \%$ & $-5.5 \%$ & $3.0 \%$ \\
MID & $1.3 \%$ & $0.1 \%$ & - & - \\
INS & $5.6 \%$ & $0.0 \%$ & $3.5 \%$ & $-1.2 \%$ \\
\hline
\end{tabular}

only [6]. A TAC was obtained from a whole-lung region of interest (ROI) which had been eroded away from edges to avoid effects of motion. A TAC was also derived from the right ventricle in order to obtain an input function (IF). A 2tissue irreversible compartment model was used to determine the kinetic parameters $K_{1}, V_{b}$ and $K_{i}$. The error between the true and gated TACs and kinetic parameters were calculated.

The patient cine-CT acquisitions were amplitude gated using a PCA technique [7]. The PET data was left ungated to avoid increased noise and reflect current practice. The PET data were reconstructed with each of the three gates using a GE proprietary software for offline data processing of PET/CT data. Whole lung and aorta TACs were determined, with the latter used to obtain the patient's IF. The fractional air volumes were also calculated for each gate as before. $K_{1}$ was measured for each gate and the variations in both the whole lung TAC and this parameter determined as (max-min)/mean.

\section{RESULTS}

The fractional air volume between the extreme gates of the simulated data was varied by $8 \%$. After AC and correction for the TFE, the EXP and INS simulated TACs were found to be under- and over-estimated respectively when compared to that of the true TAC. The MID simulated TAC was negligibly different to the true. No significant difference was found between the TACs created by the two simulation methods (Figure 1). The error between the measured and true curves varied over time with the maximum error found at the peak and minimum in the tail (see table II). The errors in $K_{1}$ are presented here to represent results from all kinetic parameters and were found to be significant for all gates with a factor 1.6, 1.3 and 0.6 increase for the INS, MID and EXP respectively.

The patient fractional air volumes were found to vary by an average of $2 \%$ between the extreme gates (maximum $4 \%$ ), less than that in the simulation. The TACs for all patients were comparable in trend to the simulated data. As there is no true patient curve, a range between the three gates was determined with the patient average found to be $7.4 \%$ and $2.9 \%$ for the

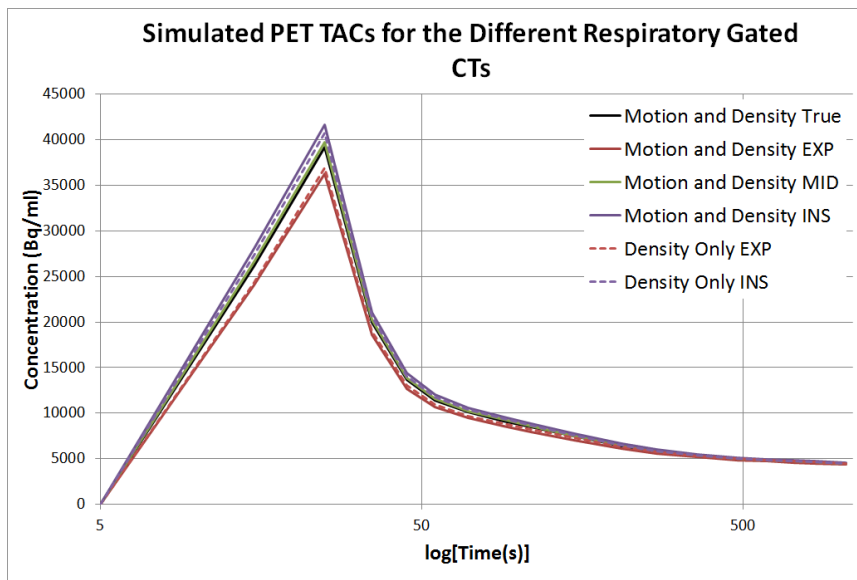

Fig. 1. Simulated TACs showing the variation between gates with negligible change between the motion and density and the motion-only case.

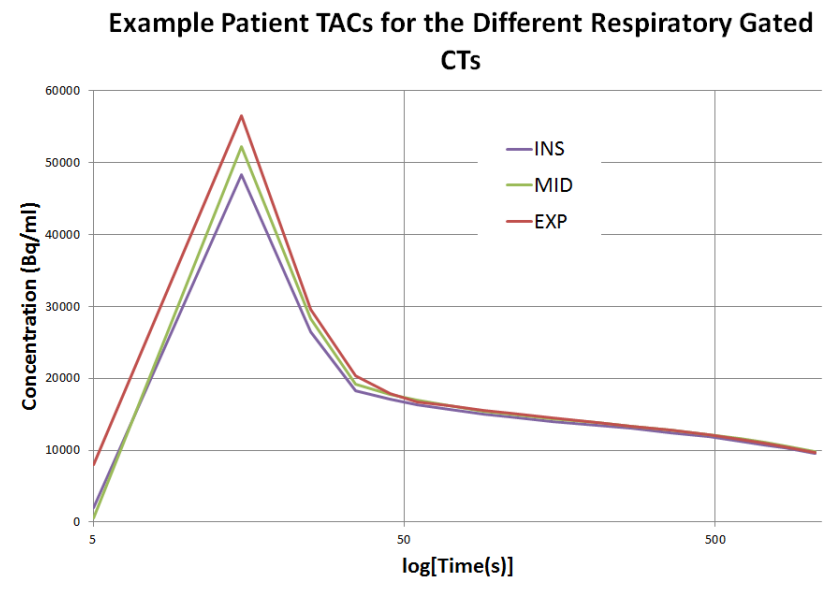

Fig. 2. Patient TACs

peak and final time point respectively. The estimates for $K_{1}$ were found to vary between gates from $3-18 \%$.

\section{DISCUSSION}

In this study we have shown that PET quantitative error varies with both density and tracer distribution leading to significant changes in the TACs and therefore the estimated kinetic parameters. The simulations found a more significant error on the parameter estimates with respect to the patients. This is due to IPF causing the patients to have shallow breathing with respect to the phantom data, displaying the dependence of the error on the extent of the density variation. Density variation in healthy individuals is known to be as high as $20 \%$, leading to higher errors in patients with a normal degree of lung expansion during respiration.

The dependency on uptake distribution, as shown by the varying error in the TACs over time, suggests that the resulting error will vary with radioactive-tracer, most likely leading to greater errors with lower pulmonary uptake.

Dynamic whole lung imaging is becoming more popular for use in studies of patients suffering from, for example, chronic obstructive pulmonary disease, intersitial lung disease, infection and inflammation. Obtaining accurate kinetic parameters 


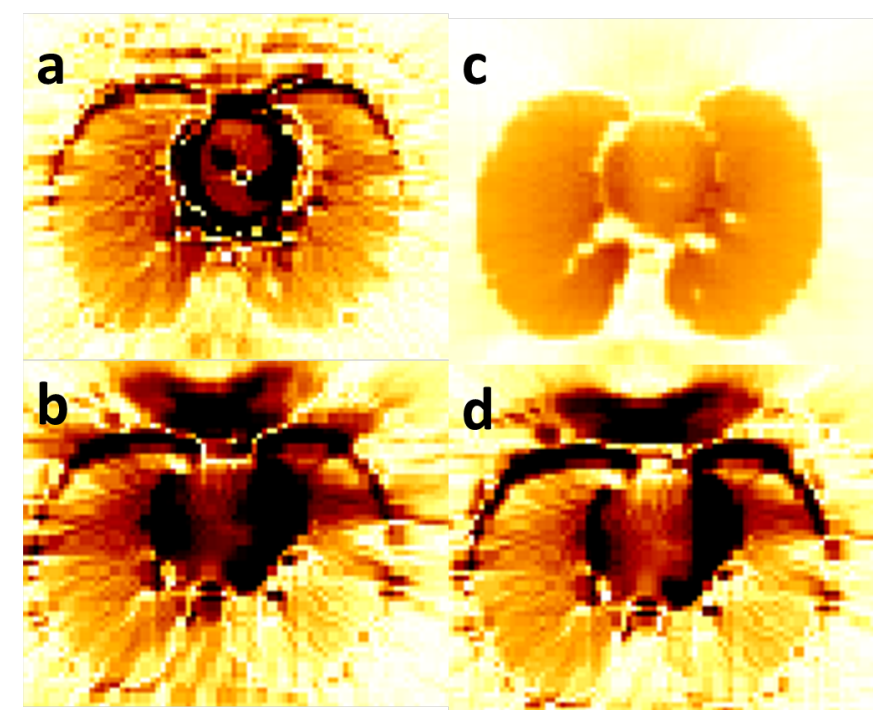

Fig. 3. Difference images between the expiration gate and the true XCAT images at the peak and end time frames. Images $a$ and $b$ are the density change only and density plus motion cases for the peak time frame respectively. Images $\mathrm{c}$ and $\mathrm{d}$ are the density change only and density plus motion cases for the final time frames respectively. It is clear from these images that the quantitation differences in the lungs are associated with the density changes. Colour scale in images $\mathrm{a}$ and $\mathrm{b}$ are -3000 to $3000 \mathrm{~Bq} / \mathrm{ml}$ with 0 displayed in white. Colour scale in images c and d are -1000 to $1000 \mathrm{~Bq} / \mathrm{ml}$ with 0 displayed in white. The significant reduction in the difference at the final time point displays the effect for the tracer distribution on quantitation error.

is required in these studies for drug development, survival studies and determination of pathophysiology. It is therefore vital to obtain a matched attenuation map for accurate PET quantitation when reviewing the whole lung or any region of the lung.

\section{CONCLUSION}

Consideration of the effects of respiratory motion are widely explored. However, little attention is given to the whole lung density mismatches which lead to inaccurate PET quantitation even away from edges and high density gradients. The extent of the errors induced by density mismatches is dependent both on the lung fractional air volume and the tracer uptake distribution at the time of measurement. It is therefore important that the CT matches the PET, in location and density, to ensure quantitative accuracy.

\section{REFERENCES}

[1] K. Thielemans, E. Asma, R. M. Manjeshwar, A. Ganin, and T. J. Spinks, "Image-based correction for mismatched attenuation in pet images," Nuclear Science Symposium Conference Record, 2008. NSS '08. IEEE, pp. 5292-5296, 2008.

[2] M. Teras, T. Tolvanen, J. J. Johansson, J. J. Williams, and J. Knuuti, "Performance of the new generation of whole-body pet/ct scanners: Discovery ste and discovery vct," Eur J Nucl Med Mol Imaging, vol. 34, no. 10, pp. 1683-92, 2007.

[3] W. P. Segars, G. Sturgeon, S. Mendonca, J. Grimes, and B. M. Tsui, "4d xcat phantom for multimodality imaging research," Med Phys, vol. 37, no. 9, pp. 4902-15, 2010.

[4] T. Lambrou, A. M. Groves, K. Erlandsson, N. Screaton, R. Endozo, T. Win, J. C. Porter, and B. F. Hutton, "The importance of correction for tissue fraction effects in lung pet: preliminary findings," Eur J Nucl Med Mol Imaging, vol. 38, no. 12, p. 2238, 2011.
[5] B. Holman, D. Butler, A. Groves, and B. Hutton, "Improving pulmonary tissue fraction correction of pet images: Is correcting for collagen and blood required?" J. Nucl. Med., vol. 54 (Supplement 2 Meeting Abstracts), no. 2 , p. 2078,2013

[6] K. Thielemans, C. Tsoumpas, S. Mustafovic, T. Beisel, P. Aguiar, N. Dikaios, and M. W. Jacobson, "Stir: software for tomographic image reconstruction release 2," Phys Med Biol, vol. 57, no. 4, pp. 867-83, 2012.

[7] K. Thielemans, S. Rathore, F. Engbrant, and P. Razifar, "Device-less gating for pet/ct using pca," Nuclear Science Symposium and Medical Imaging Conference (NSS/MIC), 2011 IEEE, pp. 3904-3910, 2011. 